volume where $\frac{P \sigma}{p_{0}}=\mathrm{I}$ and $\mathrm{x} / n$ has its ustal significance-the slope of the curve. Hence our adsorption equation for the system silica gelsulfur dioxide would be,

$$
V=0.1038\left(\frac{P_{\sigma}}{P_{0}}\right) 0.447
$$

where $V$ is expressed in cubic centimeters, $\sigma$ in dynes/cm., and $p$ and $p_{0}$ in the same unit of pressure. The close agreement is very striking and is strong evidence of our clain that the volume occupied by the adsorbed vapor is the same at the same value of the corresponding pressure $p / p_{0}$.

Summary.

I. The adsorption of sulfur dioxide by silica gel was measured at various temperatures between $-80^{\circ}$ and $+100^{\circ}$.

2. The effect of the water content of the silica gel was studied. Maximum adsorption was shown by gels containing about $7 \%$ water.

3. The adsorption was shown to be reversible in the absence of air. In the presence of small amounts of air the rate of adsorption was greatly decreased and adsorption and desorption were irreversible.

4. The empirical equation of Freundlich was found to hold over almost the entire range studied-exceptions being at these points where the saturation pressure was approached.

5. The equation

$$
\frac{V}{\sigma^{1 / n}}=K\left(p / p_{0}\right)^{1 / n}
$$

is found to hold, where $V=$ volume of condensed phase uncorrected, $\sigma$ the surface tension, $p$ the pressure of the gas phase, $p_{0}$ the vapor pressure of the liquid, $k$ and $\mathrm{r} / n$ constants dependent upon the physical properties of the adsorbent.

BALTLMORE, MARYLAND.

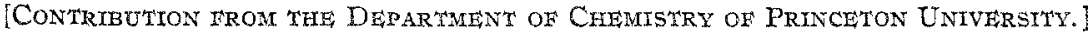

\title{
A. SURFACE CONDENSATION ERROR IN CERTAIN MEASURE- MENTS OF VAPOR PRESSURE BY THE GAS CURRENT SATURATION METHOD.
}

By ALAN W. C. MENZIES.

Recejved March 20, 1920.

In the employment of the method referred to in the title, the possibility of condensation of the saturated vapor on surfaces earlier in the apparatus train than the weighed absorption tubes has been appreciated by some investigators but neglected by others. In order that the seriousness of this source of error in the case of water may be more fully understood, the writer proposes to illustrate its incidence in certain investigations 
and to report the results of experiments that give an idea of the magnitude of the error in the case of glass and of asbestos surfaces.

Saturated Water Vapor and the Gas Laws.-It may be well to clear the ground by stating that the assumptions that even the saturated vapor of water in air at ordinary temperatures and pressures is of normal density as computed from a molecular weight of 18.016 and a standard gram molecular volume of 22.40 liters by the gas laws, and that Dalton's law is followed by the mixture, seem to be justifiable within the limits that would be indicated by the following experimental results: vapor pressure of water in mm. mercury at $0^{\circ}$ by static method, according to Scheel and Heuse, ${ }^{1}$ at $25^{\circ}, 23.76$; at $30^{\circ}, 31.83$; vapor pressure by air saturation method, employing the above assumptions, at $25^{\circ}, 23.7$ (Lincoin and Klein $\left.{ }^{2}\right) ; 23.7 \mathrm{I}$ (Krauskopf ${ }^{3}$ ); 23.75 (Derby, Daniels and Gutsche $\left.{ }^{4}\right)$; at $30^{\circ}, 31.80\left(\right.$ Perman $\left.^{5}\right)$. The fact that such agreement continues good throughout a range of higher temperatures ${ }^{6}$ makes it unlikely that the coincidence is due to a balancing of errors. It may here be noted that no one of these workers reports the use of glass wool, asbestos or other plugs to fiter their saturated air stream. The work of Galizine ${ }^{7}$ points to the close applicability of Dalton's law at higher temperatures and lower total pressures for water and other vapors.

Erratic Results in Measurements of Vapor-Pressure Lowering.-For purposes of molecular weight determination, W. Ostwald suggested the consideration of the weights of vapor of solvent lost by potash bulbs containing respectively ( $\mathrm{I}$ ) solution of non-volatile solute of known concentration and (2) pure solvent, when the same current of gas was passed through each in series. The development of this apparently simple method, which has not even yet been brought to a form suitable for everyday laboratory application, furnishes a most interesting history.

Walker ${ }^{8}$ carried out the method for the solvent water, and states explicitly that cotton-wool or asbestos fings, introduced to catch possible suspended droplets, worked "moce harm than good." Will and Bredig" applied Walker's technique to alcoholic and ethereal solutions, and promised to develop the method fu-ther. That they had not done so in the interim did not deter Orndorff and Carroll ${ }^{10}$ from making the effort, some

${ }^{3}$ Ann. Physik., 3I, 73I (r9ro).

2 J. Phys. Chem., II, 318 (1907).

s Ibid., $\mathrm{x}_{4}, 489$ (19ro).

a This Journal, 36,793 (1914).

5 Proc. Roy. Soc., 72, 72 (1903).

${ }^{-C f}$. Perman, loc. cit.

'Ann. Physik., 4I, 588, 770 (I89o).

${ }^{8}$ Z. phys. Chem., 2, 602 (x888).

${ }^{9}$ Ber, 22, 1084 (I889).

10 J. Phys. Chem., $x, 753$ (1897). 
8 years later, to adapt the method to laboratory practice. In discussing erratic results, they sense the presence of some disturbing factor other than defective saturation of the air current, and suggest surface tension. They promise further work. Speyers, ${ }^{1}$ who wished to determine molecular weights in water and other solvents, frankly abandoned his unsuccessful attempts to use this procedure. But the method is full of fascination, and Carveth and Fowler, ${ }^{2}$ in 1904 , again made the attempt to improve the technique and render the results reliable. They are surprised that this method of molectlar weight determination has not found favor with organic chemists, as it involves only the most common of laboratory appliances, and they suspect that such others as attempted it with unsatisfactory results must have failed to publish. Using a series of 3 water saturator tubes of Benedict's type ${ }^{3}$ containing glass wool, and later, glass beads, they were disconcerted to find the second saturator tube gaining instead of losing weight in both instances, while the third, control tube, which should have remained constant, lost much more than the second gained. The reasons for this behavior should become clear in the light of the experimental results recorded below, and one can well agree with Carveth and Fowler that the anomaly does not necessarily arise from unequal temperature. They feared especially that saturation had not been attained by previous workers. Although they took pains to guard against this source of error, they were much dissatisfied with their results, and state that "the subject may be taken up at a later date." As in the case of the other workers, however, they do not seem to have published further results.

In regard to the completeness of saturation of an air-current of reasonable speed by water vapor under the experimental conditions that have obtained with most workers, it may be stated that the observations of Perman, ${ }^{4}$ Lincoln and Klein ${ }^{4}{ }^{4}$ Krauskopf ${ }^{4}$ and others are altogether reassuring. The difficulty has, in reality, lain, not in saturating the gascurrent, but in keeping it saturated until it entered the absorption vessel.

Washburn and Heuse ${ }^{5}$ report the use of no glass wool or other filter between saturator and absorber. They evaporated and condensed over to g. of water in a 24 -hour run, and obtained $0.5 \%$ concordance. As is obvious from the curves below, the transfer of such large weights of water tends to diminish greatly the error due to premature condensation. Their apparatus is somewhat elaborate.

Erratic Results in Measurements of Dissociation Pressure.-The gas current saturation method has not hitherto had a very wide applica-

I J. Phys. Chem., r, 766 (1897).

${ }^{2}$ Ibid., $8,3 \times 3$ (1904).

"Am. Chem. J, 23, 326 (romo).

${ }^{4}$ Lot. cit.

"THes JOUknat, 37,309 (1915). 
tion in this field, and it is, therefore, gratifying to observe the very recent studies made by Baxter and Lansing ${ }^{2}$ of the use of this method. The writer also hopes to report his own procedure in the near future. Surface condensation of vapor is less to be feared when the vapor is not sacturated, and reference will, therefore, be made only to the work of Partington ${ }^{2}$ and Tammann. ${ }^{3}$ Each of these workers passed the same gas current through (1) powdered salt hydrate, (2) absorption tubes, (3) ptre water, and (4) again absorption tubes, and, from the relative weights of vapor absorbed, obtained the relation between the dissociation pressure of the salt hydrate and the vapor pressure of water at the same temperature. The surface absorption error is here likely to be incident to the gain in weight of (4).

In 2 series of experiments on the same salt hydrate, barium chloride dihydrate, Partington obtained average results differing by $4.6 \%$. Partington appreciates the discrepancy, but offers no explanation of it other than a mention of "irregularities." Scrutiny of Partington's description and diagram of his apparatus reveals the utilization of a plug of glass wool between the water saturator and the water absorber, which was not weighed with the latter. As the 2 series of experiments were apparently separated by a considerable interval of time, the simple explanation, in the light of the data given below, is that the content of water on the surface of the glass wool had changed in the meantime. This explanation is the more probable by reason of the very small total weights of water that passed over the glass wool-less than 4 centigrams for the sum of the earlier series of 7 experiments-in marked contrast to the use of over ro g. per experiment, with no glass wool, in the work of Washburn and Heuse.

The lack of uniformity in Tamman's rest1ts may have other causes, ${ }^{4}$ but he employed an asbestos filter for his air-current of $100 \%$ humidity. Asbestos is a very much less uniform material than even glass wool, so that it is hard to establish a normal behavior for it that would apply to past performances of other samples. It is hoped, however, that the simple findings reported below may serve to remind other workers to be on their guard in using it in similar cases.

Condensation of Water Vapor on Glass Surfaces.-References to the literature of this subject may be found in the writings of Freundlich, ${ }^{5}$ Pettijohn $n^{6}$ and others. The investigators there referred to, however, were especially careful to clean their experimental glass surfaces, whereas those workers who have used glass wool plugs incidentally to gas current

i TuIs JOURnAL, 42, 4 r9 (1920).

'J.Chem. Soc., 99, 466 (1911).

s Ann. Physik., 33, 322 (1888).

"Ci. Campbell, Trans. Faraday Soc., ro, r97 (19r4).

"Kapillarchemie 177, 265 (1909).

is ThIS JOURNAL, 41, 477 (1919). 
saturation measurements refer to its use only casually, and in no case give details as to its history, character or treatment. With a view, therefore, to explaining such anomalous results as those referred to above, it appeared to be desirable to obtain data as to the fraction of the weight of saturated water vapor passing over it that is taken up by "ordinary" glass wool, and also by asbestos, under the customary conditions of such experiments.

Experimental Procedure.-Much depends on what treatment a sample of glass wool must receive in order that it shall be considered representative. Some might reason that, for the present purpose, and in the absence of any statement by the users as to special treatment, glass wool was normal and representative of its kind simply as received from the dealers. Even Drucker and Ullman, in a research on the surface effect of glass in vapor density determinations, do not mention washing or steaming their glass wool, but merely drying it. For the present work, however, what was done was to place the glass wool in a small calcium chloride tube, to treat it for ro minutes with a rapid current of wet steam at $100^{\circ}$, to wash it very thoroughly with distilled water, and finally to dry it at $250^{\circ}$ for Io minutes in a current of air. In view of the findings of Sherwood," it was judged unfair to dry at higher temper" ature. The glass wool was of recent American purchase, and of unknown history and kind. Of the two samples available, "coarse". and "fine" respectively, the coarse variety was selected.

A specimen was prepared for measurement by approved though laborious sampling methods, and the diameters of 30 fibers measured under a microscope with eyepiece micrometer whose readings were evaluated by a stage micrometer ruled directly on glass. Photographically reproduced stage micrometers are often several per cent. in error, and, in view of the wide variation of fiber sizes and consequent unavoidable uncertainty of average diameter, it was especially undesirable to inject any gratuitous source of error. The average diameter was $0.00308 \mathrm{~cm}$, with a "standard variation" of $0.00084 \mathrm{~cm}$., giving the mean error of the mean as $0.000 \mathrm{I} \mathrm{cm}$. The diameter of the glass wool fibers used by Drucker and Ullmann ${ }^{3}$ was $0.0019 \mathrm{~cm}$; by Parks, ${ }^{4} 0.00 \times 75 \mathrm{~cm}$.; and by Leech and the writer in $1912,0.0032 \mathrm{~cm}$.

Assuming that all the surface of the glass wool is cylindrical and that the density of the glass is 2.60 , one may compute that the surface of the glass wool used was about $650 \mathrm{sq}$. cm. per gram. The weight of glass wool used was $1.837 \mathrm{~g}$, and the total glass surface within the calcium cluloride tube was thus about I $230 \mathrm{sq} . \mathrm{cm}$.

2. phys. Chem., 74, 567 (xgro).

2 Thus Journat, 40, I645 (6918).

${ }^{3}$ Loc. cit.

\&Pil. Mag., [6] 5, 5 I7 (1903). 
Air saturated with water vapor at $22^{\circ}$ was aspirated through this glass wool tube, and the issuing water vapor collected in weighed absorption tubes. In saturating the air, it was especially necessary to avoid bubbling and consequent possible air-suspended droplets. For this reason, saturators somewhat of the Kahlenberg type were utilized, and the cotton wool filtered air was aspirated over the surface of distilled water that half filled 3 horizontal tubes in series, each exposing a surface about $5 \times 25 \mathrm{~cm}$, kept practically stagnant. Thence the air passed into a conical flask, containing a few mm. depth of distilled water, above which the glass wool tube was supported. All 4 vessels were completely submerged in a very well-stirred water thermostat tank. A number of runs were made, each lasting from 3 to 30 hours; and from the gains in weight of glass wool tube and absorption tubes were computed in each case (A) the percentage of the total water vapos concerned in each run that was retained by the glass wool tube, $(B)$ the weight of water, in mg. per sq. cm. of glass surface, present at the middle of each run, and (C) the weight of water vapor in $\mathrm{g}$. that had been concerned since the beginning of the first run until the middle of the run in question in building up the water found in $B$.

Experimental Findings. Case of Glass Wool.--The results of 8 runs are tabulated below, using the above notation:

\section{TABLE I.}

Condensation of Saturated Water Vapor on Glass Wool at $22^{\circ}$.

\begin{tabular}{|c|c|c|c|c|}
\hline Run No. & $\begin{array}{l}\text { Liters } \\
\text { per hour. }\end{array}$ & A. & $\begin{array}{c}\text { B. } \\
\text { mg. } / \mathrm{cm}^{2} \text {. }\end{array}$ & $\mathrm{G}$. \\
\hline$I \ldots \ldots \ldots \ldots$ & 3.8 & 13.9 & 0.012 & 0.106 \\
\hline $2 \ldots \ldots \ldots \ldots$ & I. 9 & 4.56 & 0.038 & 0.597 \\
\hline $3 \ldots \ldots \ldots$ & I.O & $2.7 x$ & 0.056 & 1.29 \\
\hline $4 \ldots \ldots \ldots$ & $7 \cdot 4$ & $x .59$ & 0.076 & 2.67 \\
\hline $5 \ldots \ldots \ldots$ & $\operatorname{I\pi } .8$ & 0.92 & 0.095 & 4.45 \\
\hline $6 \ldots \ldots . .$. & 5.8 & 0.84 & 0.107 & 6.11 \\
\hline $7 \ldots \ldots \ldots$ & $9 \cdot 7$ & 0.79 & 0.118 & $7 \cdot 77$ \\
\hline $8 \ldots \ldots \ldots \ldots$ & 4.0 & 0.79 & 0.127 & 9.15 \\
\hline
\end{tabular}

Fig. I shows these results graphically in 2 curves, $A B$ and $B C$, in which the values of $B$ are abscissas in both cases. As the points observed fall reasonably well on smooth curves, it is evident that the speed of the air current, within the limits tabulated, exerts little influence on the results. No comment is necessary on the magnitude of the errors to which in this case, surface condensation is thus seen to give rise, for the figures speak for themselves. The film of condensed water in Run 8 is obviously about 1.27 microns thick, and can hardly be regarded as ats "adsorbed" layer in the usual sense.

An additional datum may be drawn, in confirmation, from the careful

1 Science, July 21 , I905. 
work of Berkeley and Hartley. In their earlier measurements of the vapor peessure from water, these observers invariably found that their absorption tube gained less water vapor than was lost by the tubes supplying the vapor-by $0.37 \%$ in an experiment at $19^{\circ}$ which they quote as typical. In their still more elaborate later work, ${ }^{2}$ they sought to minimize the amount of this troublesome discrepancy, and were led to

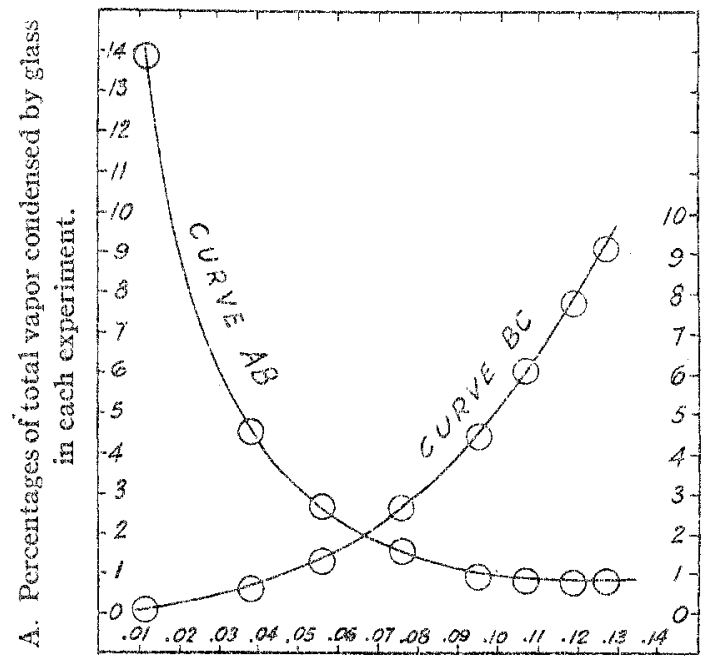
adopt a removable form of connecting tube, to join the water saturator with its absorption tube. This connecting tube could thus be weighed for itself, and invariably gained weight in each experiment. In the only example cited, carried out at $30^{\circ}$, the tube gained $0.17 \%$ of $2.84 \mathrm{~g}$. of saturated water vapor that passed through B. Milligrams condensed water per square crn. glass surface. it in 24 hours. EstiFig. I.-Relation of weight of water condensed on glass to mating, from the ex(A) and (B). cellent drawing, the area of the inner surface of the tube at ro sq. cm., one would obtain an abscissa of $0.48 \mathrm{mg}$. for a point on curve $B C$ with an ordinate of 350 . It is well worth remarking that such skilful workers, using no glass wool and dealing with considerable weights of water vapor, are seriously but unavoidably inconvenienced by the condensation of saturated water vapor on the inner walls of a carefully cleaned short plain glass connecting tube.

Case of Asbestos.-As already indicated, it appeared hopeless to obtain what might be regarded as an average sample of asbestos on account of the enormous irregularity in several respects of various samples. A specimen of acid-washed asbestos "for Cooch crucibles" was steamed for 30 minutes, soaked in water overnight, washed with distilled water and dried in its calcium chloride tube in an air current with gentle heating. Comparing the weights of water condensed by one gram of asbestos and of glass wool on treatment at $22^{\circ}$ with 0.63 and $2.44 \mathrm{~g}$. of saturated water vapor respectively, the asbestos took up $32 \%$ and $28 \%$, respectively, as much water as, according to curve $\mathrm{BC}$, would be taken

'Proc. Roy Soc. (London), 77, 156 (1906).

2 Trans. Roy. Soc. (London), 209A, I77 (Igog). 
up by each gram of glass wool under like conditions. This points to a qualitative agreement in behavior of asbestos and glass wool.

\section{Summary.}

The condensation of water from its saturated vapor in air on a steamed and water-washed glass surface and on an acid-washed, steamed and water-washed asbestos surface has been investigated under the conditions that have prevailed with many users of the gas-current saturation method of vapor pressure measurement. The amount of this condensation has been found to be sufficiently great to largely account for unexplained irregularities recorded in the literature of measurements by this method.

FRINCETON, N. J.

[CONTRIPUTION FROM THE DAPARTMENT OF CHEMISTRY OF CORNELL UNIVARSTTY.]

\section{A SEARCH FOR AN ALKALI ELEMENT OF HIGHER ATOMIC WEIGHT THAN CESIUM.}

By L. M. Dennis and R. W. G. Wyckofr.

Received March 22, 1920.

Ass a result of his study of the $\mathrm{X}$-ray spectra of the elements from aluminum to gold, Moseley ${ }^{1}$ assigned atomic numbers to the elements, beginning with $\mathrm{I}_{3}$ for aluminum and closing with 79 for gold. He found known elements to correspond with all of these numbers except 3 , and hence considered that there are probably 3 undiscovered elements between aluminum and gold. Siegbahn and Friman ${ }^{2}$ extended the work of Moseley to the series of elements from tantalum to uranium, and found 3 unfilled places between polonium, atomic number 84 , and radium, atomic number 88 . The element with an atomic number of 87 would lie to the left of radium and would fall in Group I under cesium.

Search for this element, which may provisionally be termed eka-cesium, has already been made by Richards and Archibald," and by Baxter." Richards and Archibald subjected $150 \mathrm{~g}$. of cesium dichloro-iodide to fractional crystallization with the object of concentrating eka-cesium, if it was present, in the fractions at one end of the series. Baxter fractionally crystallized a large amount of cesium nitrate. Neither investigation gave indication of the presence of a higher analogue of cesium.

It seemed desirable that further search for this eka-cesium be made, and that there be employed methods of fractionation involving the use of salts of cesium showing greater differences of solubility than do the

i Phir. Mag., 27, 703 (1914).

2 Ibid., 32, 39 (1916).

${ }^{3}$ Proc. Am. Acad., 38, 443 (1903).

4THIS JoURNAr, 37, 286 (I915). 